$16^{\text {th }}$ International Congress of Metrology, 06011 (2013)

DOI: $10.1051 /$ metrology/201306011

(c) Owned by the authors, published by EDP Sciences, 2013

\title{
National comparison on verification of fuel dispensers
}

\author{
Ana Almeida ${ }^{\mathrm{a}}$, Nelson Almeida, Elsa Batista, Eduarda Filipe, Cartaxo Reis \\ IPQ - Instituto Português da Qualidade, Rua António Gião, 2, 2829-513 Caparica, Portugal
}

\begin{abstract}
In order to check the agreement of results and procedures in the field of verification of fuel dispensers by the verification bodies designated by the Portuguese Institute for Quality (IPQ), a national comparison between these entities was performed for the first time.

A correction for reference temperature was performed to the results presented by the participants, maximum flow at $20 \mathrm{~L}$ and minimum flow at $5 \mathrm{~L}$, using petrol and diesel as verification liquid.

The analysis of the results, using the normalized error statistics, evidenced satisfactory values, for the majority of the national entities. The uncertainty components and values were very similar within the participants for the minimum flow at $5 \mathrm{~L}$. For higher volume, the uncertainties were very dependent on the type of volume standard used.
\end{abstract}

\section{Introduction}

Like any kind of measuring instruments used for commercial transactions, fuel dispensers, commonly known as petrol pumps, are submitted to metrological control in order to assure consumer protection and provide to society in general and citizens in particular the guarantee of accurate measurements.

The Portuguese Institute of Quality (IPQ) is the National Body for Legal Metrology and defines the general rules of legal metrology, prepares metrological regulation, co-ordinates the metrological control activities, performs patterns approval and verifications and designates notified bodies and verification bodies.

The legal metrological control in Portugal is widely decentralized in 768 verification bodies among governmental, regional and local authorities, public and private companies.

The designation process of the verification bodies is based on NP EN ISO/IEC 17020 and NP EN ISO/IEC 17025. The majority of the 54 national verification bodies are accredited laboratories and certified companies (NP EN ISO 9001) and are involved in the verification of 25 different specific measuring instruments. One of these measuring instruments is the fuel dispenser.

In Portugal the fuel dispensers approved under the MID are subject to metrological control in service, thought its annual verification by the verification bodies, following internal procedures, according to specific national regulations [1].

Actually there are 45000 hoses of fuel dispensers in Portugal, verified by several verification bodies equally distributed in the national territory.

\subsection{Comparisons in legal metrology}

Accredited laboratories often perform comparisons in order to verify the agreement of results and procedures. In legal metrology however this is not a usual practice. IPQ decided to implement the practice of organizing comparisons between designated verification bodies for legal metrological control. The first chosen metrological field was the verification of fuel dispensers and six verification bodies decided to participate, although using different equipment and experimental procedures.

The comparison was piloted by IPQ that prepared the protocol, including all the experimental details and a form sheet for the results, sent to all participants before the beginning of the comparison. As pilot laboratory, IPQ performed measurements before and after the ones of the participating laboratories, providing the reference value and its associated uncertainty.

The comparison was performed during one week. Each participant used their own volume standards and had 2 hours to make the on-site measurements.

\subsection{Fuel dispensers}

The fuel dispenser (figure 1) used in the comparison was supplied by a national company and had the following characteristics:

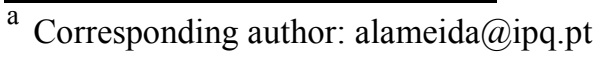


Table 1. Fuel dispenser characteristics

\begin{tabular}{|c|c|}
\hline Brand & PETROTEC \\
\hline Model & P5000 \\
\hline Class & $\begin{array}{c}0,5 \text { according to the } \\
\text { National Regulation } n^{\text {o }} \\
19 / 2007,5^{\text {th }} \text { of January }\end{array}$ \\
\hline Resolution & $0,01 \mathrm{~L}$ \\
\hline Engraving & MID CE M11 0866 \\
\hline Diesel hose & $\mathrm{N}^{\mathrm{o}} 3$ \\
\hline Petrol hose & $\mathrm{N}^{\mathrm{o}} 5$ \\
\hline
\end{tabular}

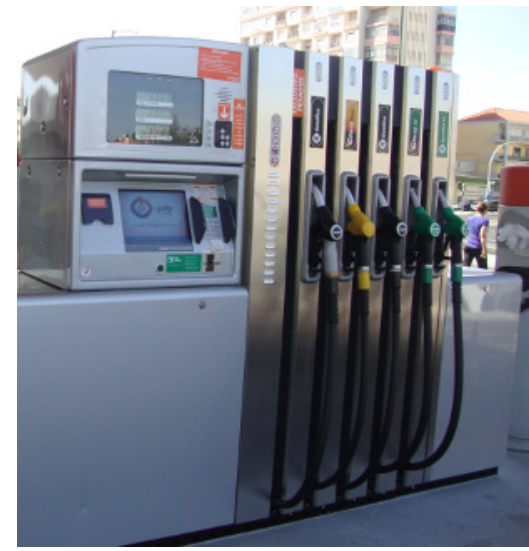

Figure 1. Fuel dispenser used in the comparison

\section{Experimental procedure}

The experimental procedure was included in the protocol sent to the participants.

Each participant had to verify the delivered volume of the fuel at maximum flow using a $20 \mathrm{~L}$ volume standard and the delivered volume of the fuel at minimum flow using a $5 \mathrm{~L}$ volume standard. The verification liquids used were petrol and diesel, by this order due to residual volume increase. Five measurements were performed for each volume standard using the two different fuels.

The variation of maximum allowable temperature for the liquid was $0,5^{\circ} \mathrm{C}$ during the five measurements. The ambient conditions had to remain between the following intervals: the temperature between $15^{\circ} \mathrm{C}$ and $35^{\circ} \mathrm{C}$, the relative humidity between $25 \%$ and $75 \%$ and pressure between $840 \mathrm{hPa}$ and $1060 \mathrm{hPa}$ [2].

The volume standards were calibrated by recognized entities and the delivery time respected [3].

Each participant was asked to use the normal work routine, meaning the operator, the procedure and the equipment.

For the sake of confidentiality, the names of the participant entities are not presented and replaced by codes, for example PE1 (Participant Entity 1).
The characteristics of the volume standards used by each participant are presented in figures 2 and 3 and in table 2:

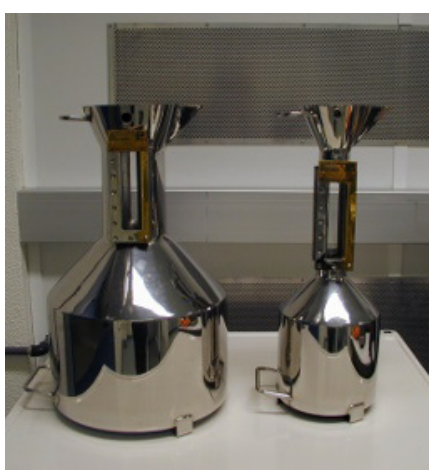

Figure 2. Stainless steel volume standard

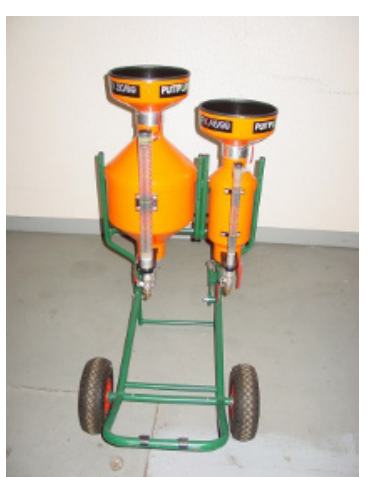

Figure 3. Carbon fiber volume standard
Table 2. Characteristics of the volume standards used by the different participants

\begin{tabular}{|c|c|c|}
\hline Participants & Type & Resolution \\
\hline IPQ & Carbon Fiber & $\begin{array}{l}0,01 \% \text { for the } 20 \mathrm{~L} \\
\text { standard; } 0,02 \% \text { for } \\
\text { the } 5 \mathrm{~L} \text { standard }\end{array}$ \\
\hline PE1 & $\begin{array}{c}\text { Carbon } \\
\text { Fiber }\end{array}$ & $\begin{array}{l}0,01 \% \text { for the } 20 \mathrm{~L} \\
\text { standard; } 0,02 \% \text { for } \\
\text { the } 5 \mathrm{~L} \text { standard }\end{array}$ \\
\hline PE2 & Stainless steel & $0,05 \%$ \\
\hline PE3 & Stainless steel & $0,10 \%$ \\
\hline PE4 & Stainless steel & $0,5 \%$ \\
\hline PE5 & Stainless steel & $0,10 \%$ \\
\hline PE6 & Stainless steel & $\begin{array}{l}0,10 \% \text { for the } 5 \mathrm{~L} \\
\text { standard; } \\
0,05 \% \text { for the } 20 \mathrm{~L} \\
\text { standard }\end{array}$ \\
\hline
\end{tabular}

\section{Statistical analysis}

\subsection{Reference value determination}

The reference value used was the mean value of the two measurements performed by IPQ, the first one at the beginning and the other at the end of the comparison. The uncertainty of the reference value considered was the largest value of both measurements.

\subsection{Results analysis}

The results analysis was performed according to the $E_{n}$ number $[4,5]$ : 
$E_{\mathrm{n}}=\frac{\left(V_{\mathrm{EP}}-V_{\mathrm{Ref}}\right)}{\sqrt{U_{\mathrm{EP}}^{2}+U_{\mathrm{Ref}}^{2}}}$

Where $V_{\text {ep }}$ and $U_{\text {ep }}$ are the volume and the corresponding expanded uncertainty obtained by the participant entity and $V_{\text {ref }}$ and $U_{\text {ref }}$ are the volume and the corresponding expanded uncertainty obtained by the reference laboratory (IPQ).

According to normative documents, absolute values of $E_{n}$ smaller or equal than 1 represent a satisfactory performance of the participant entity.

\section{Measurement results}

In order to obtain comparable measurement results they were corrected to a reference temperature of $20{ }^{\circ} \mathrm{C}$ using the standard model [6]:

$$
V_{20}=V_{t}[1+\gamma(20-t)]
$$

Where, $\gamma$ is the cubic thermal expansion coefficient of the volume standard material and $t$ the liquid temperature.

\subsection{Volume determination}

The volumes obtained by the participants using diesel and petrol at a maximum flow $(20 \mathrm{~L})$ and minimum flow $(5 \mathrm{~L})$ are presented in the following figures (fig. 4 to fig. 7):

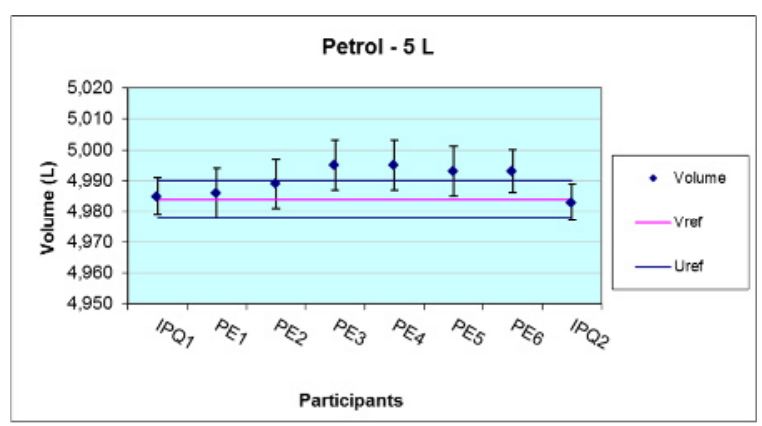

Figure 4. Delivered volume for petrol at minimum flow

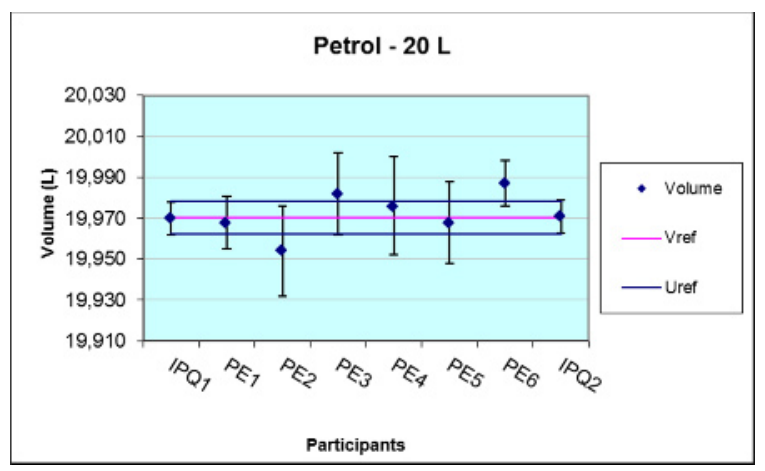

Figure 5. Delivered volume for petrol at maximum flow

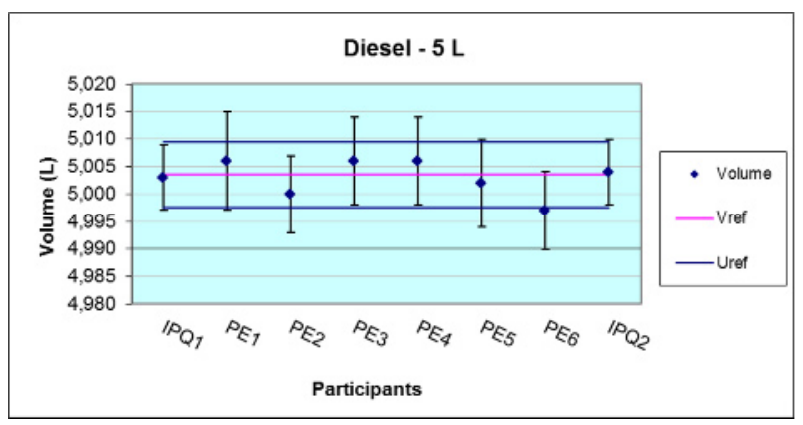

Figure 6. Delivered volume for diesel at minimum flow

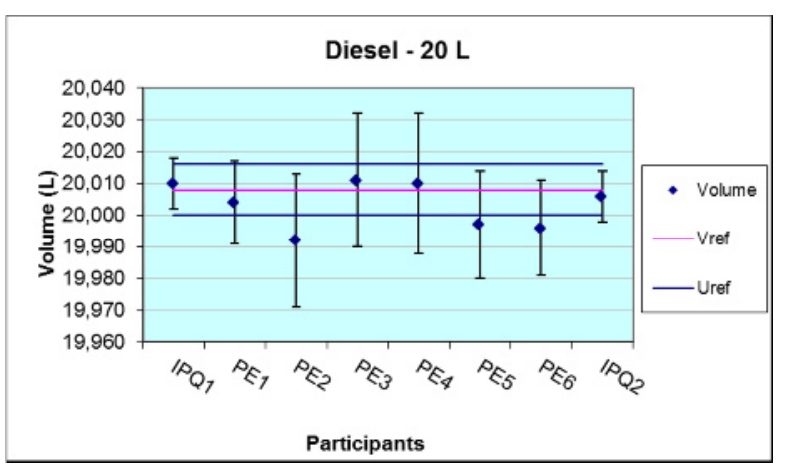

Figure 7. Delivered volume for diesel at maximum flow

The majority of the results are very similar and the variation between participants is smaller than $0,2 \%$ which is less than the maximum permissible error (MPE) of these instruments. Indeed and according to the Portuguese national regulation [1] and the Measuring Instruments European Directive [7] the MPE for fuel dispensers is $0,5 \%$. The worst cases are for petrol using the $5 \mathrm{~L}$ volume standard, which was expected, due to the volatility of the fuel and the small quantity of fluid delivered.

\subsection{Normalized error}

The normalized errors, $E_{n}$ numbers as defined previously, obtained for the measurement results of each participant entity, are displayed in the figures 8 to 11 .

Normalized Error - Petrol 5 L

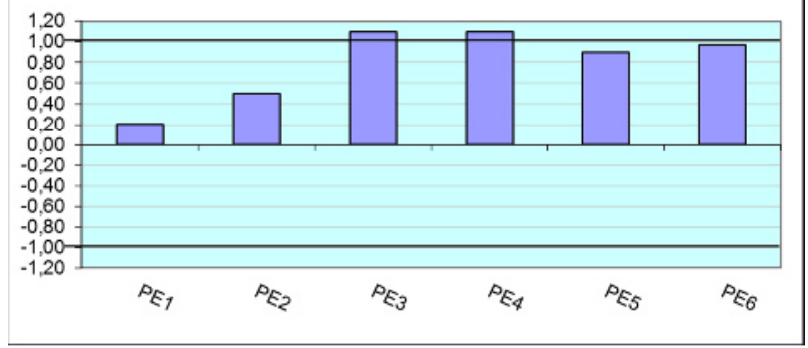

Figure 8. Normalized error for petrol at minimum flow 


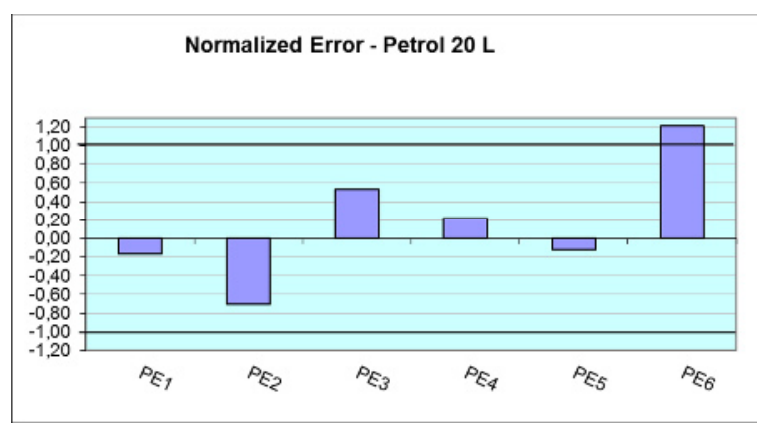

Figure 9. Normalized error for petrol at maximum flow

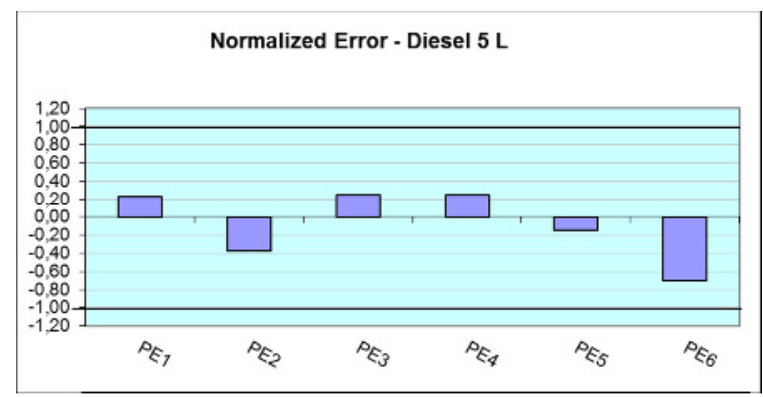

Figure 10. Normalized error for diesel at minimum flow

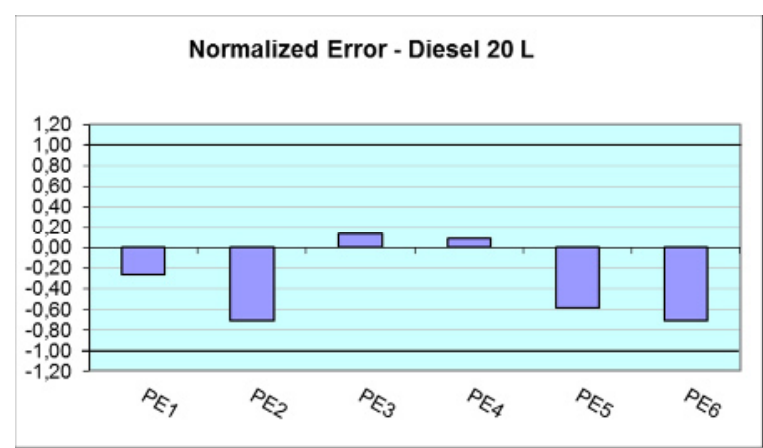

Figure 11. Normalized error for diesel at maximum flow

Inconsistent results are only found for petrol and mainly for $5 \mathrm{~L}$, but very close to 1 . A possible explanation is that the participants did not respect the order of the liquids described in the protocol, first petrol and then diesel. This behavior leads to a higher volume than the reference value due to the extra residual volume.

\section{Uncertainty calculation}

When starting the comparison, a spread sheet was supplied for the presentation of the results and the uncertainty budget according to the "Guide to the Expression of Uncertainty in Measurement" [8].

In order that each participant results could be compared on the same ground, some common uncertainty components to be included at the uncertainty budget were suggested by the pilot laboratory. These uncertainty components were: the repeatability of the measurements ( $u$ rep), the calibration of the volume standard ( $u$ cal vs), the expansion coefficient of the volume standard ( $u$ exp coef), the resolution of the volume standard ( $u$ res vs), the meniscus reading ( $u$ men), the resolution of the fuel dispenser ( $u$ res fd) and the liquid temperature ( $u$ temp). Almost all participants evidenced uncertainty budgets according to the pilot suggestions.
The results for $5 \mathrm{~L}$ and $20 \mathrm{~L}$ using petrol are displayed in figure 12 and in figure 13. As the results for diesel are very similar, they are not presented.

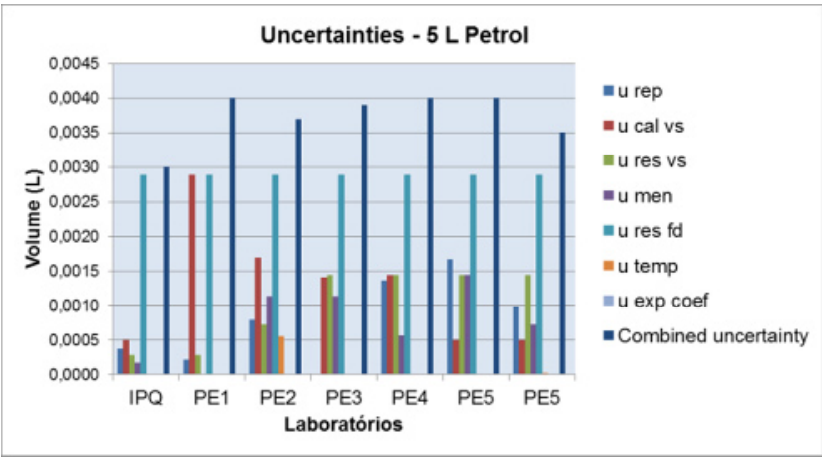

Figure 12. Uncertainty for petrol at minimum flow

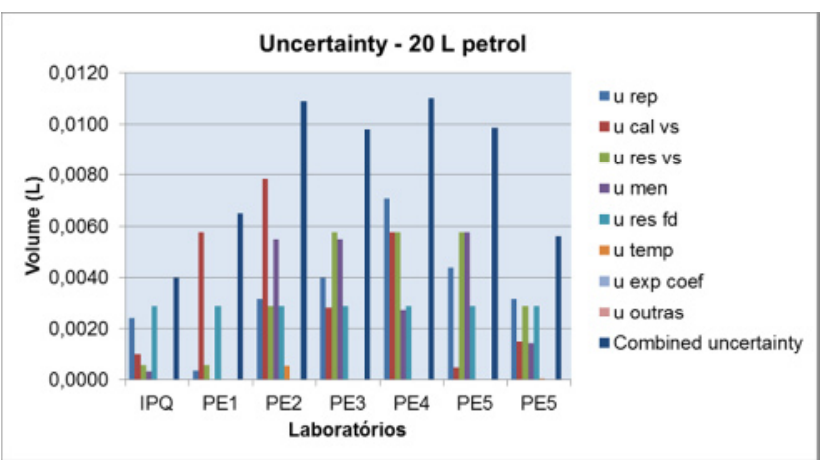

Figure 13. Uncertainty for petrol at maximum flow

For $5 \mathrm{~L}$, the largest source of uncertainty is the resolution of the fuel dispenser and the expanded uncertainties are very similar for all participants.

For $20 \mathrm{~L}$, it is not possible to make the same conclusion regarding the largest contribution of the uncertainty component. The value of the combined uncertainty is different between the participants. The reference laboratory and one the participants used a volume standard with better resolution of the scale and consequently better uncertainty - carbon fiber volume standard.

\section{Conclusion}

For the first time, a national comparison in field of verification of fuel dispensers was organized between 6 qualified entities for metrological control. The IPQ-NMI has piloted this comparison, providing the reference value.

Considering that the measurements were performed on-site, at different days and hours, by different entities using volume standards with different characteristics, the results of the comparison can be seen as positive.

Although three participants had unsatisfactory results in the petrol measurements, their $E_{n}$ values were very close to 1. Such situations occurred because the participants did not comply with the order of the liquids as described in the protocol, which stated that petrol should be used before diesel. Indeed, higher volume than the reference value was always obtained if the protocol was not followed. 
Interestingly, the volume values measured by the participants had a maximum variation between each other of $0,2 \%$. This value is smaller than the MPE of the fuel dispensers and indicates a good reproducibility of the whole measurements.

Concerning the evaluation of the measurement uncertainty, we can verify that, for $5 \mathrm{~L}$ with both kinds of fuels, the largest source of uncertainty of all participants was the resolution of the device under test. For $20 \mathrm{~L}$, there was no evidence regarding the largest source of uncertainty and correspondingly the value of the expanded uncertainty was very different among the participants.

\section{References}

1. National regulation $n^{\text {o }} 19 / 2007,5^{\text {th }}$ of January

2. OIML R117-1 - "Dynamic measuring systems for liquids other than water, (2007)

3. N. Almeida, E. Batista, E. Filipe, "Evaluation of the influence of the liquid used in the verification of fuel dispensers and their standard calibration”, OIML Bulletin L, 4, (2009)

4. ISO/IEC 17043:2010 - Conformity assessment General requirements for proficiency testing

5. ISO 13528:2005 - Statistical methods used in proficiency testing by interlaboratory comparisons

6. ISO 4787:2010 - Laboratory glassware Volumetric glassware - Methods for use and testing of capacity

7. DIRECTIVE 2004/22/EC of the European Parliament and of the Council, of 31 March 2004, on measuring instruments

8. "Evaluation of measurement data - Guide to the expression of uncertainty in measurement" JCGM 100:2008 (GUM 1995 with minor corrections) 